\title{
Toward Dynamics, Stability and Processing of The Vision System
}

\author{
Hooshang Hemami ${ }^{1} \&$ Vadim I. Utkin ${ }^{1}$
}

\author{
${ }^{1}$ Dept. of Electrical and Computer Engineering, The Ohio State University, Columbus, Ohio 43210, USA \\ Correspondence: Hooshang Hemami, Dept. of Electrical and Computer Engineering, The Ohio State University, Colum- \\ bus, Ohio 43210, USA. E-mail: hemami.1@ osu.edu
}

Received: September 2, 2019

Accepted: October 10, 2018

Online Published: November 30, 2019

doi:10.5539/mer.v9n2p1

URL: https://doi.org/10.5539/mer.v9n2p1

\begin{abstract}
Eye movements are vitally important for living, survival and vision. A basic model of the eye, including ocular muscles, is considered that may be essential for better understanding the optical and sensory apparatus involved in vision, perception, and processing and control machinery of human movement. A state space model based on Newton-Euler formulation of the dynamics of the head and the eyeballs is presented that can be integrated with the dynamics of the skeletal system. The formulation allows integration of Lyapunov stability, hypothesized feedback mechanisms and inverse dynamics in more comprehensive neural processing models. Neural networks involved in saccades, gazes and dynamics of specific injuries, diseases and defects can be included in this formulation. The development allows a better understanding of the vision system as well as the neural and physiological elements involved in perception and physical and mental skills. It is also relevant to the design of artificial eyes.
\end{abstract}

Keywords: eyeball dynamics, linear models, ocular muscles, Lyapunov stability, artificial eyes

\section{Introduction}

A six-body system is envisioned here that involves three neck elements, the head and the two eyeballs. There are passive structures around the eyes and six ocular muscles for each eye. The two eyeballs are inside the head, do not have large weights and are not subjected to external forces, stresses and dislocation. On the other hand, the ocular muscles are not accessible for easy studies and measurements. The muscles of the neck lie on top of each other (Basmajian, 1978) and are involved in rotations of the head, movements of the neck, speaking, breathing, swallowing, coughing and perhaps other human activities. We will consider simple muscular structures, not necessarily corresponding to an accurate physiology and function of the actual neck muscles. This allows computer simulations of the simpler functions of the neck and easier movements of the head.

Two classes of simple point-to-point human movements are taking a step and moving the eye (or eyes) in a gaze or saccade motion. These two movements are different in one important respect. Stepping is subject to external conditions and disturbances. Gaze and saccade movements take place in the head and are almost protected from all disturbances. Stepping and many other locomotory movements require elaborate feedback mechanisms of the spindles for stability (Szentagothai and Arbib, 1974). While mathematical models of stepping systems are highly nonlinear and require Lyapunov and higherlevel stability studies, gaze and saccade do not have feedback mechanisms which may cause difficulty with computer simulations. The computer model may have to be stabilized with artificial mechanisms that do not interfere with the physiological behavior of the system and do not distort and interfere with the real movement that is being simulated.

This issue will be a challenge to future modeling, analysis and understanding of the central nervous system (CNS). The computer programs need stabilization mechanisms that would not disturb or distort the actual behavior of the CNS.

Another central difficulty with monitoring movements is how they are observed, extracted from the environment, stored in memory and recalled later. One model (Hemami et al., 2016b) uses roll, pitch and yaw as rotational states of all rigid bodies of the skeleton. For the eyes, the ocular muscles can be modeled as pairwise being activated in the coordinate system of the head in the inertial coordinate system (ICS). Each of the three components of movement can be stored in stacks of position and velocity (Hemami et al., 2016b) or in phase plane trajectory of the velocity versus position (Brooks, 1986, p. 119). Optical feedback is not considered here.

A more complex movement without spindle or negative feedback may be the movement of the lower lip and jaw collaborating in speech generation (Brooks, 1986, p. 164).

The saccade motion of the eyeballs is a relatively fast movement and has special attributes. Other fast human movements 
are somersault and motion in the air (Hemami et al., 2016a). Ballistic movements are similar (Hemami and Dariush, 2016). Physical and control structure of marionettes have similar properties (Hemami and Dinneen, 1995).

Many studies deal with smooth pursuit, saccade and gaze (Becker and Juergens, 1975; Brooks, 1986, Rogers, 2011). The ocular muscles perform five different functions ((Kandel et al., 1991), Chapter 43). They are the vestibulo-ocular, optokinetic reflexes, saccades. smooth pursuit and vergence. The geometry and physiology of the muscle models here are consistent with anatomy (Gray, 1977; Boileau Grant., 1962, Barin, 2009). The masses are small. Large muscular forces, involving stretch reflexes, (Winters, 1990) are not involved. Therefore, apparently, different muscle physiology may be involved.

From a system theoretical point of view, the major components of the vision system are rigid body dynamics, including the muscular system, stability of the system, and CNS processing and control of the eyes. The integration of these systems, computer simulations and comparison of the simulations with experimental, anatomical and diseased structures are left for future work. Systems of three - and four - rigid bodies have been formulated before (Hemami, 2003; Hemami and Wyman, 2012; Hemami et al., 2016b). Therefore, a head and three neck segments could be modeled as a four rigid - body system. The sliding of the neck bones on each other may use ligamental control as passive control, similar to such activity at the knee joint (Wongchaisuwat et al., 1984; Hemami et al., 1987). A linear model of the muscular system is constructed that allows independent study of the roll, pitch and yaw of each eyeball.

The ocular muscle system is represented by two models. One is a simple and non-real system that facilitates stability, input design, and activation of motion by pairs of muscles (Hogan, 1984). The second one is more realistic and has approximate geometry and topology of average human eye muscles. The stability of the simpler ocular system can be carried out and demonstrated by the construction of Lyapunov functions in a systematic way, analogous to the methods of construction of such functions for linear stable time-invariant systems (Khalil, 1996; Utkin, 2009). The procedure is detailed in (Humphrey, 2009; Hemami and Utkin, 2002). This analysis is carried out without feedback due to stiffness reflex of ordinary muscles. Finally, it is shown how the central nervous system (CNS) may use the linear knowledge to adapt to nonlinear and time-varying case of construction of inputs to the eye muscles.

Alternative methods of analysis and control are adaptive methods (Kokotovic and Arcak, 2000) and singular perturbation methods for fast dynamics of the eyeballs and slow dynamics of the head (Kokotovic et al., 1986; Khalil, 1996). The rotation of the eyeballs in the head is analogous to the head rotation in the ICS. Therefore, the head coordinate system (HCS) behaves as an ICS for the eyes. Cartesian positions and velocities constitute six states for each eyeball

The Euler angles and the angular velocities of the eyeballs (Hemami, 1982; Hemami and Katbab, 1982) are the body coordinate system (BCS) of the right eye (RCS) and the left eye (LCS).

In the following, dynamics and the equations of motion are presented in Section Two. Stability and the Lyapunov method are presented in Section Three. Supporting mechanisms and neural processing are discussed in Section Four. Discussions and conclusions follow in Section Five.

\section{Dynamics and the Equations of Motion}

The equations of motion of the head and the eyes are presented here The objective is to include the head and the eyeballs as rigid bodies in the musculoskeletal model of the human. The head can be represented by standard state space formulation of rigid bodies. Two movements are considered here in some detail: smooth pursuit and ballistic fast movements (Hannaford and Lehman, 1986; Hemami and Barin, 2017; Kandel et al., 1991). The eye saccades (Hannaford and Stark, 1985) are interpreted as fast movements of the eyes, similar to fast movement of the head (Hannaford and Stark, 1984). At the same time, eye movements are different than the head (or body) movements in several ways:

- no external and or variable loading.

- small inertias, and friction.

- small amplitudes and excursions.

As a consequence, and to a first order of approximation, one can assume that the eye transients are fast and negligible and can be ignored. This means the system follows central inputs accurately. Therefore, a major component of central inputs is the design of kinematic signals that are responsible. A point of hope here is the availability of experimental, medical and diseased (Bahill and Stark, ) data available from internal and external injuries, handicaps and natural failures.

\subsection{The Head}

According to Basmajian (Basmajian, 1978), the movements of the neck that cause combinations of translation and rotation of the head are flexion, extension, bending and rotation. In terms of rigid body dynamics terminology, flexion and 
extension correspond to pitch, bending to roll and rotation to yaw movement. To further clarify the terminology, the roll axis is orthogonal to the frontal plane. The pitch axis is orthogonal to the sagittal plane. The yaw axis is orthogonal to the transverse plane.

The muscles of the neck are involved in many movements. The semi-spinalis is almost limited to one main function, namely pitch motion of the head. The splenius capitis is active in pitch and roll. Motion of the neck and arms are related in sternomastoid and scalenes action. Some of the muscles of the neck also have anti-gravity functions. The point of discussion here is that the neck and head muscles are involved in many movements and physical activities. Also, monitoring the muscles for identification of physical and neural activity is not feasible. One approach, adopted here, is to use individual muscle components dedicated to specific action and perhaps combine the actions and muscle components later in more accurate physical and neurally realistic muscle models.

To keep the present analysis simple, it is assumed that the three segments of the neck are stationary in the inertial coordinate system (Hemami, 2002a). The head's coordinate system is centered at the point of contact of the head with the neck. The three Euler angles are roll, pitch and yaw (Hemami, 2003). Let the coordinates of the center of gravity of the head in the HCS system be $C_{0}$, given in Eq.1.

$$
C_{0}=[0,0, c]^{\prime}
$$

The head is vertical at its equilibrium point above the neck. When the head moves, it can be an inverted pendulum connected to the neck. Therefore the center of gravity of the head is lower than its position at the equilibrium.

Let $\Theta_{h}$ and $\Omega_{h}$ be, respectively, the Euler angles and the angular velocity vector of the head expressed in the head coordinate system (HCS). Let $A$ and $B$ be the standard state space matrices. Let vector $G$ be the gravity expressed in the ICS. Let $N_{h}$ be the sum of the moment of force vectors, expressed in ICS. $N_{g}$ is the torque of gravity (?). $N_{a}$ is the anti-gravity torque afforded by part of the same muscles involved in moving the head or by a separate set of anti-gravity muscles. Let the torque of the three pairs of muscle forces, involved in moving the head be $N_{f}$. Let $G$ be the gravity vector expressed in ICS. The expression for the total torque $N$ is given by Eq. 2

$$
\begin{array}{r}
G=-M g[0,0,1]^{\prime} \\
N_{f}=[B]^{T} K_{c}\left(\Theta_{d}-\Theta\right)-L_{o}(\Omega) \\
N_{g}=[\breve{C}] A^{T} G . \\
N_{a}=-N_{g} \\
N_{h}=N_{f}+N_{g}+N_{a}
\end{array}
$$

In the above equation, the control strategy of gravity compensation (afforded by the vestibular system), position feedback (afforded by the stretch reflexes) and velocity feedback ( due to linear friction, reflex, etc.) are utilized. The equations of motion of the head (Hemami and Katbab, 1982) are given in Eq.3

$$
\begin{array}{r}
\dot{\Theta}_{h}=B\left(\Theta_{h}\right)\left(\Omega_{h}\right) \\
J \dot{\Omega}_{h}=\breve{\Omega}_{h} J\left(\Omega_{h}\right)+N_{h}
\end{array}
$$

The components of the neck are stationary in the inertial coordinate system (Hemami, 2002a). The head's coordinate system is centered at the point of contact of the head with the neck. Let the coordinates of the center of gravity of the head in the HCS system be $C_{0}$ given in Eq. 1. The head is vertical at its equilibrium point above the neck. When the head moves, it can be an inverted pendulum connected to the neck. Therefore the center of gravity of the head is lower than its position at the equilibrium.

Ballistic movements of the head are similar to other human ballistic movements (Hemami and Dariush, 2016). These movements are fast and require tri-phasic central inputs (Hannaford and Stark, 1985). The control and neural machinery of the ballistic movements of the head are similar to movements of the arms (Hemami and Dariush, 2016). Four CNS structures are involved in trajectory design.

- Basal ganglia in intensity and gain control (Hemami and Moussavi, 2014). 
- Reticular formation may be involved in timing (Hemami and Dariush, 2016).

- The cerebellum may be involved in coordination.

- The hippocampus may involved in processing of stored trajectories ((Kandel et al., 1991), Chapter 1) and may appear to be involved in motivational issues and emotions (Hemami, 2017) and ((Angevine Jr and Cotman, 1981), Chapter 12).

For lack of precise knowledge about dynamics of the CNS, one may have to model and program components of the CNS as best as one can program them and interface them with a musculoskeletal system that is physically better understood.

\subsection{The Eyeballs}

It is assumed that the eyeballs are spherical, and their moment of inertia matrix is diagonal with three equal elements. Consequently the nonlinear term:

in Eq. 3 disappears.

At the equilibrium position of the right eyeball, the RCS and HCS are aligned with HCS, which is aligned with ICS: axis $x$ for roll is to the front, axis $y$ for pitch to the left and axis $z$ for yaw upwards. All muscles are stretched to be along the axes of the HSL. All the six attachment (insertion) points of the muscles are on four points in the RCS, i.e., in the $y z$ plane. The radius of the eyeball is about $12 \mathrm{~mm}$. In the model we assume a radius of unity. A simple six-muscle model is presented for the eyeball that decouples the three motions of roll, pitch and yaw at the equilibrium point. For the decoupling function, it is assumed that the inferior oblique and superior oblique (numbered 1 and 2) pair of muscles have the same insertion coordinate and rotate the eye, respectively, in positive and negative $\left(\theta_{1}\right)$ directions. The two unit vectors that define the direction of the two muscular forces, are, respectively, in the $y$ and $-y$ directions.

The pitch motion of the eyeball, at the equilibrium, is affected by a torque produced along the $y$ axis by the pair of inferior and superior rectus muscles (numbered 3 and 4 ). The insertion points are at $[0,0,-1]^{T}$ and $[0,0,1]^{T}$ on the eyeball. Both forces for these muscles are in the negative $X$ axis direction.

The yaw motion (in the $x y$ plane) is affected by the pair of muscles: medial rectus for positive $\theta_{3}$ and lateral rectus for negative $\theta_{3}$. The insertion points are, respectively, $[0,1,0]^{T}$ and $[0,-1,0]^{T}$. The direction of forces is along the negative $x$ axis as the previous two muscles

This decoupled muscle system may help the design of actual control mechanisms and would be a first step in the design of artificial eyes.

Let the magnitude of all six forces be unity. With the above model, the six unit vectors $u$, the corresponding insertion point $a$ and the resulting torques $\tau$ are

- inferior oblique for positive roll $\mathrm{i}=1$

$$
u=[0,-1,0]^{T}, a=[0,0,1]^{T}, \tau=[1.0 .0]^{T}
$$

- superior oblique for negative roll $\mathrm{i}=2$

$$
u=[0,-1,0]^{T}, a=[0,0,1]^{T}, \tau=[-1.0 .0]^{T}
$$

- inferior rectus for positive pitch $\mathrm{i}=3$

$$
u=[-1,0,0]^{T}, a=[0,0,-1]^{T}, \tau=[0,1,0]^{T}
$$

- superior rectus for negative pitch $\mathrm{i}=4$

$$
u=[-1,0,0]^{T}, a=[0,0,1]^{T}, \tau=[0,-1,0]^{T}
$$

- medial rectus for positive yaw $\mathrm{i}=5$

$$
u=[-1,0,0]^{T}, a=[0,1,0]^{T}, \tau=[0,0,1]^{T}
$$


- lateral rectus for negative yaw $\mathrm{i}=6$

$$
u=[-1,0,0]^{T}, a=[0,-1,0]^{T}, \tau=[0,0,-1]^{T}
$$

Let the magnitude of the exerted force by muscle $i$ be $q_{i}$. The moment of force applied to the eyeball by muscle $i$ is

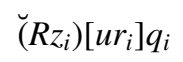

Based on the above model, the six torques can be projected along the three eyeball axes: roll, pitch and yaw as the following vector:

$$
\begin{aligned}
& N 1=0.012\left(q_{1}-q_{2}\right) \\
& N 2=0.012\left(q_{3}-q_{4}\right) \\
& N 3=0.012\left(q_{5}-q_{6}\right)
\end{aligned}
$$

The friction effects of the eyeball moving in the eye socket can also be taken into consideration by assuming a linear model: a diagonal 3 × 3 matrix of equal elements multiplied by $\Omega$.

As a consequence, and to a first order of approximation, one can assume that the eye transients are fast and negligible and can be ignored. This means the system follows central inputs accurately. Therefore, a major component of central inputs is the design of kinematic signals that are responsible. A point of hope here is the availability of experimental, medical and diseased (Bahill and Stark, ) data available from internal and external injuries, handicaps and natural failures.

Before presenting the above issues, it is important to establish that the dynamics of the eye, in terms of stability and control exerted by the muscular system, are equivalent to the dynamics of the muscular system of the head. This subject merits a thorough analysis and is postponed for a separate effort.

A second issue, in this regard, is the physical geometry and attachment of the eyeball muscular insertions and origins and their relevance to specific motions and eye trajectories. Higher-level neural circuits are always involved. Another important issue from system theoretical and neural points of view is the issue of constraints. Due to the attachment constraints, the motion of the eye in the RCS or LCS are coupled. For specific motions along the different axes there is need for decoupling or alternatively, projecting the desired motion as combination of other physically available motions.

The eyeballs and the ocular muscles, on the other hand, are better understood as rigid bodies and sources of force. The position vectors of the origins of all the 12 ocular muscles, the trochlear hook of both eyes, and the centers of the two eyeballs are specified in HCS. The equations for the two eyeballs are similar to Eq.3. The inertial coordinate system for both eyes is the HCS. The vector $\mathrm{N}$ for each eyeball has two components. One component is due to the action of the six muscles to be described below. The second component is the small friction force when the eyeball moves. Let $\Omega$ be the angular velocity vector of the eyeball. The friction moment of force is modeled as

$$
N=-N_{f} \Omega
$$

where $N_{f}$ is a diagonal positive $3 \times 3$ matrix and $\Omega$ is the angular velocity of the eye in HCS.

We order from 1 to 6 the six muscles of the right eyeball: superior oblique, inferior oblique, inferior rectus, superior rectus, medial rectus and lateral rectus. The insertion points of the six muscles can be specified by vectors $R_{i}$ in the right eye coordinate system (RCS). Let the unit vector, tangent to the eyeball and in the direction of the force of the muscle be $u_{i}$ Let the magnitude of the force of muscle $i$ be $q_{i}$. Finally, as discussed in (Hemami, 2002b), let the antisymmetric $3 \times 3$ matrix associated with $S$ be $\breve{S}$.

In general $u_{i}$, and the lengths of the six muscles depend on the position of the eyeball in the head and hence are functions of the Euler angles of the eyeball: $\Theta$. The moment of force of muscle $i$ on the eyeball is

$$
\left(q_{i}\right) \breve{R}_{i}\left(u_{i}\right)
$$

The total moment of force is the sum of the moments of the six muscles. We notice that, to the extent that these muscular forces, as specified, depend on the Euler angles of the eye, they define a structural (mechanical) feedback component. 
Vectors $R_{i}$ are specified in the eyeball coordinate system as $R z_{i}$ at

$$
\Theta=[0,0,0]^{\prime}
$$

. As the eyeball moves,

$$
R_{i}=A(\Theta) R z_{i}
$$

The unit vectors in the direction of the muscular forces and possible involvement of the lengths of the eye muscles in the muscle force could introduce feedback that could help stability. These could be counted as negative feedback, and their role in linear systems is well known. However, their significance in saccades is not verified (Collins, 1975). Stability of the above simple model near the equilibrium point of the eyeball is studied here by the Lyapunov method in some detail.

Similar dynamics can be constructed for the left eyeball.

\subsection{Motion of the Eye}

Smooth pursuit eye movements can use visual feedback for stability purposes. These feedbacks replace standard stretch reflexes (Kandel et al., 1991, Chapter 37) and guarantee stability of the multi-linkage system of the head or the head and the body (Hemami and Hemami, 2016).

Ballistic movements of the eyes do not require stretch reflexes. They, however, require reference input signals to the eyeball system. These reference signals come from subjecting the head to the same ballistic movement without moving the eyes. The three angular accelerations of the head (roll, pitch and yaw) are sensed in the ballistic head movement, integrated twice with respect to time (by LTPs), and stored in permanent memory. The reference trajectories of position, velocity and acceleration for the corresponding eye ballistic movement are permanently available on demand (Hannaford and Stark, 1985; Hemami and Barin, 2017). To simplify the presentation, we take the simple model presented above and design the central controller for an active saccade or a counter-saccade that counteracts the head's fast movement. The model is assumed to have natural points of origin and insertion Therefore, the dynamics can be described by three secondorder linear differential equations as shown in Eq 5.

$$
J \ddot{\Theta}=-L \dot{\Theta}+Q_{a} U_{a}+Q_{b} U_{b}
$$

where $U_{a}$ and $U_{b}$, respectively, define the vectors of odd and even muscular forces

$$
\begin{aligned}
& U_{a}=[u 1, u 3, u 5]^{T} \\
& U_{b}=[u 2, u 4, u 6]^{T}
\end{aligned}
$$

The $3 \times 3$ matrices $J$ and $L, Q_{a}$ and $Q_{b}$ are as follows. Matrix $J$ is diagonal with three equal moments of inertia of the eyeball. Matrix $L$ is diagonal with three equal friction coefficients. Matrices $Q_{a}$ and $Q_{b}$ define the coefficients of the six forces of the ocular muscles in Eq. 7.

$$
\begin{aligned}
Q_{a} & =\left[\breve{R}_{1} R_{1}+\breve{R}_{3} R_{3}+\breve{R}_{5} R_{5}\right] \\
Q_{b} & =\left[\mathrm{R}_{2} R_{2}+\breve{R}_{4} R_{4}+\breve{R}_{6} R_{6}\right]
\end{aligned}
$$

In the simple model, the two matrices $Q$ will be identity matrices. So when natural origin and insertion points are assumed, the first function of the CNS ( i.e, the cerebellum in this case) has, by learning, to construct the inverses of $Q_{a}$ and $Q_{b}$ in the neural paths to the muscles. It can be shown that the cerebellum can also help with the real-time linearization of the system. This latter fact may improve the accuracy of the processing and the accuracy of the computational behavior.

The above role of the cerebellum is consistent with previous models of the feed-forward control of the ocular muscles as discussed in (Angevine Jr and Cotman, 1981), page 203, figures 4 and 5. The inputs to the cerebellar processor are the 12 ocular muscle forces (Howard, 1982), page 198 for a desired saccade extracted from memory or vestibular accelerations of the head as the head goes through a fast intended or unintended motion. The eye motions in the latter case will counteract the head's movement (Hannaford and Stark, 1985). At the same time, With two sequential integrations by long term potentiation (LTPs) the velocities and position angles of the head also are available as inputs to the ocular system. 


\section{Stability and the Lyapunov Method}

\subsection{The Head}

To show that, with the control strategy proposed in Section 2, the head is stable, we consider the system at equilibrium by assuming that the desired position state is the equilibrium position, i.e.,

$$
\Theta_{d}=0 .
$$

For rotational stability of the head, we use position and velocity feedback as follows:

$$
N_{f}=-B^{T} K_{c} \Theta-L_{c} \Omega
$$

The Lyapunov function (Utkin, 2009; Hemami and Utkin, 2002) is constructed as the sum of the kinetic energy and the quadratic form of elastic energy stored in the muscles. Since gravity is compensated by neck muscles, it does not involve rotational stability of the head. Further, since the head is taken to be a sphere in shape, the matrices $K_{c}$ and $L_{c}$ are assumed to be positive definite symmetric matrices

$$
\left.L=1 / 2(\Omega)^{T} J(\Omega)+1 / 2(\Theta)^{T} K_{c}(\Theta)\right)
$$

Differentiating $L$ with respect to time, one obtains

$$
d / d t(L)=-(\Omega)^{T} J d / d t \Omega+(\Omega)^{T} B^{T} K_{c}(\Theta)
$$

Substitution of Eq. 9 in Eq. 10, one obtains

$$
d / d t(L)=-(\Omega)^{T} L_{c}(\Omega)
$$

Therefore $\dot{L}$ is negative and the system is stable.

3.2 The Eyeball

The objective here is to develop stability criteria for the eyeball when the head is not moving and the eyeball is in its rest position. A simplified case is presented here to introduce the main ideas. There are more basic and motivational issues involved in guiding future research in understanding higher levels of CNS, ocular and vestibular control. Suppose at and around the equilibrium point, the two unit vectors that define the agonist and antagonist recti muscles are identical. These two vectors can be approximately represented by $\operatorname{sign}(\theta)$ where $\theta$ is roll, pitch or yaw. Suppose around the origin, the magnitude of the force of the pair of muscles is the same function $q(\Theta)$. Now one constructs the diagonal $3 \times 3$ matrix $Q Q_{c}$ whose diagonal elements are $q q_{1}$ for roll, $q q_{2}$ for pitch and $q q_{3}$ for yaw. Then the action of the six muscles near the equilibrium point is represented by a feedback structure in the eyeball dynamics:

$$
\left[Q Q_{c}\right]\left[\operatorname{sign}\left(\theta_{1}\right), \operatorname{sign}\left(\theta_{2}\right), \operatorname{sign}\left(\theta_{3}\right)\right]^{T} .
$$

One can also observe that, the integral of $\operatorname{sign}(\theta)$ for all $\theta$ is $a b s(\theta)$ where 'abs' stands for the absolute value. similar to a linear full rectifier curve with a slopes of -1 and 1 .

Based on this model, the above six torques can be projected along the three eyeball axes as the following vector: Let $k$ be a scaler:

$$
k=(.012)(0.10)
$$

Let $K$ be a diagonal $3 b y 3$ matrix whose diagonal elements are $k 1, k 2$ and $k 3$. Let the position torque of the six muscles be approximated by

$$
N_{p}=-[B]^{T} K\left[\operatorname{sign}\left(\theta_{1}\right) \operatorname{sign}\left(\theta_{2}\right) \operatorname{sign}\left(\theta_{3}\right)\right]^{\prime}
$$

This result means that having six muscles with the same constant dc value at equilibrium amounts to having nonlinear finite feedback around the equilibrium point. To this position feedback torque, we add the negative linear feedback torque 
$N_{f}$ due to friction. as negative velocity feedback gain. We also need the integral of the $\operatorname{sgn}(x)$ function. Let $\mathrm{u}(\mathrm{x}) \mathrm{be}$ the unit step function, and define the absolute function ( or a linear rectifier)

$$
a b s(x)=x u(-x)+x u(x)
$$

Thus the right eyeball ,near the equilibrium, can be represented by Eq.3 and the sum of nonlinear position and linear velocity feedback

$$
N=N_{p}+N_{f}
$$

To prove that the system is stable at the equilibrium point, we construct the Lyapunov function $L((\Theta),(\Omega))$

$$
L=0.5(\Omega)^{\prime} J(\Omega)+k 1 a b s\left(\theta_{1}\right)+k 2 a b s\left(\theta_{2}\right)+k 3 a b s\left(\theta_{3}\right) .
$$

We also define, for convenience

$$
\operatorname{Sgn}(\Theta)=\left[\operatorname{sign}\left(\theta_{1}\right), \operatorname{sign}\left(\theta_{2}\right), \operatorname{sign}\left(\theta_{3}\right)\right]^{T} .
$$

Also, we know that, at the equilibrium point

$$
\Theta=[0,0,0]^{T} \text {. }
$$

It can be shown that, at the equilibrium point of the eye

$$
\dot{L}=-(\Omega)^{\prime} N_{f} \Omega-[\Omega]^{T}[B]^{T} K[S g n(\Theta)]^{T}+[S g n(\Theta)]^{T} K B \Omega
$$

The latter two terms in the above equation are equal and opposite in sign at the equilibrium. Therefore the derivative of the Lyapunov function is zero at the origin and hence the right eye is stable at the equilibrium. For the Lyapunov stability of the head, we use a similar Lyapunov function (Hemami and Katbab, 1982).

The issue of the eyeball stability near its equilibrium point has been experimentally and physiologically under some rigorous studies (Goldberg et al., 1991). There are no apparent feedback system to stabilize the eyeball near its equilibrium point. We consider the simplest possible case here. Suppose for small Euler angles of roll, pitch and yaw, the three angles are decoupled. Suppose also the main friction for the rotation is linear in angular velocity and is due to friction. Suppose also the forces of +10 gram and -10 gram that come from the pair of muscles are combined in a sign function. let the angle be $y$. The differential equation that describes the motion is

$$
J \ddot{y}+b \dot{y}+\operatorname{csign}(y)=0 .
$$

This is in contrast to the more elaborate physical models proposed (Howard, 1982), page 196, where the sign function, at zero, may also be approximated by a line with a given slope. Lyapunov methods show that both systems are stable.

There is also a philosophical issue here (Howard, 1982; ?). The implication of the involvement of matrix $B^{\prime}$ in both the head control and the eyeball control is significant. One can speculate that this matrix is the simplest, most crude and minimal model of the CNS processing in vision, vestibular and locomotion systems. Presumably, there are many varieties of $B$ in size, dependence, sophistication and versatility in the higher levels of the CNS as we begin to understand them.

\section{Supporting Concepts and Mechanisms}

The objective is to include the head and the eyeballs as rigid bodies in the musculoskeletal model of the human. The head can be represented by standard state space formulation of rigid bodies. The eyeballs, without ocular input, move with the head. Therefore, for the eyes, the head becomes their inertial coordinate system (ICS). In order to be able to visually track moving objects in the environment, the ICS positions and velocities of the eyes must be known from measurement and/or neural construction. Externally, the vision system provides such information. Internally, the positions and velocities in the ICS are constructed as the sum of the components in the head's coordinate system (HCS) and the right eye's (RCS) and left eye's coordinate system (LCS). The angular velocities $\Omega$ of the eyes can be defined in the eye coordinate systems.

For the eyes' movements, relative to the head and for the head movement in the ICS, standard rigid body state space equations hold. Further, we assume that movements of the eyeballs, relatively speaking, due to low masses and moments of inertia, do not affect the rigid body state space equations of the head. 
Let $\Theta_{h}$ and $\Omega_{h}$ define the Euler angles and the angular velocities of the head in the body coordinate system of the head (BCS). Let $\Theta_{r}$ be the angle vector of the right eye in HCS. Let $\beta_{r}$ define the angular position vector of the right eye in ICS (sensed by the vision system.) Let $\Theta_{l}$ and $\beta_{l}$ define the same quantities for the left eye Let $\Omega_{h}$ define the angle vector of the head in ICS as sensed by the vestibular accelerations and time integrators. The above angular positions and velocities for the right eye are related:

$$
\begin{array}{r}
\beta_{r}=\Theta_{r}+\Theta_{h} \\
d / d t\left(\beta_{r}\right)=B\left(\Theta_{r}\right) \Omega_{r}+B\left(\Theta_{h}\right) \Omega_{h}
\end{array}
$$

Similar relations hold for the left eye.

$$
\begin{array}{r}
\beta_{l}=\Theta_{l}+\Theta_{h} \\
d / d t\left(\beta_{l}\right)=B\left(\Theta_{l}\right) \Omega_{l}+B\left(\Theta_{h}\right) \Omega_{h}
\end{array}
$$

These relations may be involved in the dynamics of processing vision material and following moving objects and sceneries and will not be further pursued here.

To understand the vision system in the narrow sense of how it relates to human movement, one has to integrate the vision system in a computer model that can integrate all of it in one program and can modify the model as better knowledge becomes available If the physically observed subsystem does not have identifiable feedback mechanisms for stability, one may have to add artificial or extraneous mechanisms that stabilize the computer program. If these stabilizing mechanisms interfere with the behavior of the system, a practical solution may be to use inverse dynamic systems that allow replacement of the subsystem and its inverse by identity operators that simplify the design.

A second part of the design of the system may involve physical devices, concepts or components that have to be designed by standard engineering tools or programmed:

- Positive arithmetic and mathematics

- Amplitude scaling and level shifting,

- Time scaling,

- Simple oscillators, shape construction, and pattern generators, and

- Object movement and translation.

It appears, from the literature, that most of the CNS processing is carried out with positive neural signals in the range of zero to 100 millivolts. It is, therefore, desirable that the elements to be constructed and considered here all work with positive numbers and arithmetic. The modern computer works with limited signals, grossly approximated as a one and a zero. Of course, negative numbers are represented in computers. Perhaps similar mechanisms exit in the CNS for negative numbers. It is known that different amplitude ranges exist in the CNS. At the same time, most neurons have a limited amplitude range before they saturate. We consider pulse frequency modulation here for the following purposes:

- To allow transmission of larger amplitude signals by converting the amplitude to frequency of pulses that satisfy the amplitude constraint of the neuron.

- To allow scaling up or down of signal amplitudes. For DC signals, this amounts to up or down level shifting. The undesirable side effect of scaling in general is the inevitable delay introduced in the modulation, transmission and demodulation of the signal. Another side effect is that for serial consecutive multi-level scaling, the delays add. We will also investigate parallel scaling as opposed to serial scaling.

- The internal neuron structure for modulation and demodulation should be understood. 
Time scaling involves a variety of processing of a time signal. Speeding up or slowing down trajectories in time is one such process. Slowing down a signal implies stretching it linearly in time, and reducing the slopes of the derivative. Speeding up a signal means the reverse. In the frequency domain, slowing a time process involves compressing the frequency contents near zero frequency. A compression of the signal in the time domain means an expansion in the frequency domain: an increase in the bandwidth. Reversing signals in time or reflecting them in time are other time processes.

Simple oscillators to produce a repeated sequence or trajectory, shape construction, as a part of imagination or a trajectory of motion, as well as pattern generators involved in more complex motion generation or detection are examples of this more complex processing.

Finally, imaginary and real object movement, rotation and translation are additional areas we will discuss in more detail.

\subsection{Amplitude scaling and level shifting}

One mechanism for level shifting is to allow a demodulator of signals to have a natural amplification or attenuation attached to it. As an example, signals that transfer down the spinal cord and converge on motor neurons may have natural amplification relative to the source of those signals where they were modulated for transmission.

\subsection{Neural Modulation and Demodulation}

Pulse frequency modulation is thought to be used by the CNS for transmission, amplitude scaling and perhaps other purposes. A simple modulator - demodulator scheme is discussed here that identifies all the important features of the natural system. The modulator is made of the following components:

- A triggering mechanism that keeps the pulse generator off when the signal is zero.

- A basic one-shot circuit or pulse-generator that fires a three-interval signal. In the first interval the signal rises linearly to the maximum the neural axon can carry. In the second interval, the signal subsides linearly to zero. In the third interval the circuit rests. All three intervals are assumed here to have the same duration. Let the sum of the three intervals be $t$.

- A delay control mechanism that regulates the timing of when the next pulse is fired. If the input signal's magnitude is $a$, the delay is

$$
d=1-a
$$

to be modulated. Therefore the total delay between successive firings is $t+d$. When the signal has maximum amplitude, (i.e., unity here,) the delay is zero. If the amplitude is zero, the delay is assumed to be some maximum value - one second here. The process of firing is also overridden by the pulse generator or one-shot not firing. This means the delay interval is programmed to be inversely and linearly proportional to the magnitude of the input signal.

The behavior of the system is shown in two figures: Figure 1 shows a signal to be frequency modulated and transmitted.

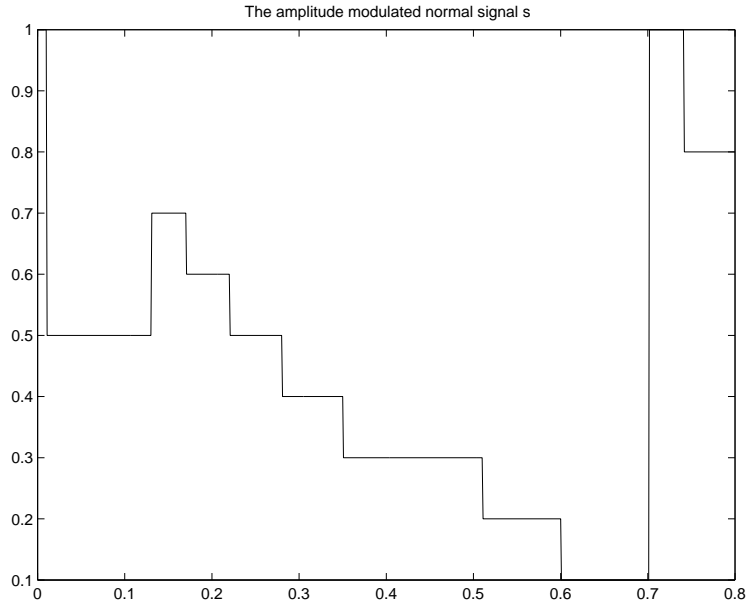

Figure 1: The amplitude-modulated normal signal s composed of 16 values 


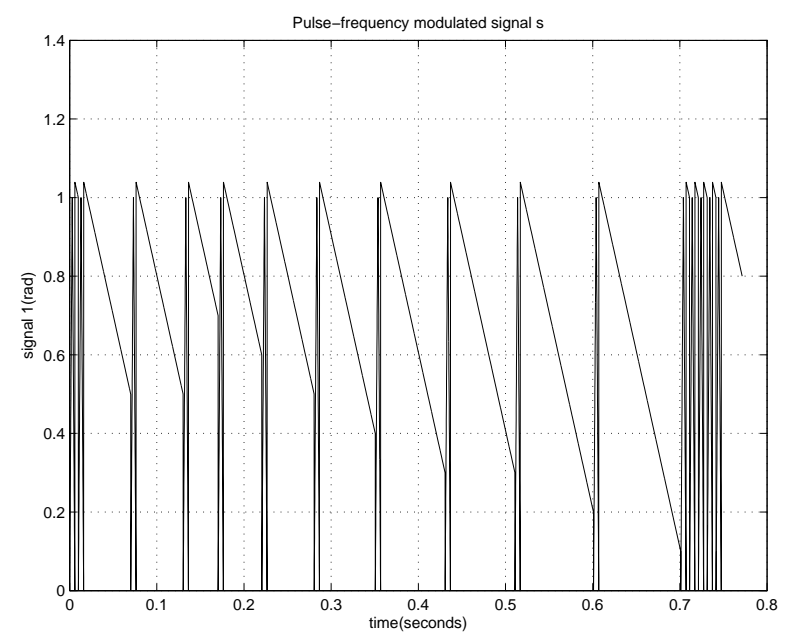

Figure 2: The output of the pulse-frequency modulator

Figure 2 shows the output of the modulator.

The latter signal is transmitted down the axon to where it is demodulated, possibly with a different threshold, i.e., it could be an amplified or attenuated version of the original signals.

The structure of the demodulator is as follows. The demodulator measures the time duration $\tau$ between successive firings. The total delay is the duration $t$ plus the delay $d$ since the delay and the amplitude are inversely related, the recovered magnitude of the signal is

$$
a=1-(\tau-t) .
$$

Therefore, at the demodulator, the total delay between the pulses is measured and leads to the magnitude of the input signal. This process results in a $100 \%$ recovery of the original signal without any loss.

\subsection{Pulse and Periodic Functions of Time}

Many signals of time are pulse shaped or are periodic. Two limitations govern these signals. The signal is always positive, and the amplitude is bound by a given maximum.

Both of these signals can be designed using the concept of FIR (finite impulse response) from the field of signal processing. With negative feedback, the pulse generator can be made into an oscillator.

\subsection{Time Scaling and Processing}

A majority of motor functions in living systems are described qualitatively, e.g., ((Kandel et al., 1991), Figs $41-14$ and 42 - 5). Most timed events and the involved neural processing of such timed events are qualitatively reported. Theoretical and system-oriented models that can be mathematically and computationally verified are desirable. Such models may also pave the way for future neural, anatomical and physiological experiments. The construction, transmission (in the CNS) and processing of reported timed events involve small numbers of neurons.

A timed event corresponds to a desired motion of a limb (Iqbal and Roy, 2009), a sequence of gains that controls production of a reflex signal ( (Kandel et al., 1991),Chapter 64) or an efferent neural signal that induces a desirable motion. Three timed events of tapping, stepping and rocking produce a longer dance maneuver (Jalics et al., 1997). Synchronizing with an external event, reversing a signal in time, and compression or expansion of signals in time are other examples. A timed event may be part of a pattern generator or an oscillator. In a dynamic system, described by an artificial neural network (ANN) or a differential equation (Bay and Hemami, 1987), a set of programmable (settable) gains control the behavior of the system as in cardiac myo-cytes (Levitan and Kaczmarek, 1991), page 250. Chemical agents and neuro-transmitters induce the needed gain changes in many instances. Serotonin is one agent known to increase the duration of action potentials in abdominal and pleural sensory neurons of aplysia ((Levitan and Kaczmarek, 1991), page 250). It inhibits and delays re-polarization due to potassium channels. Serotonin may further decrease a voltage-dependent delayed rectifying potassium current and thus prolong the action potential ((Levitan and Kaczmarek, 1991), page 254).

Internal timed events are involved in motor learning, natural reflexes, and tight input-output coupling (Evarts et al., 1971; 
?; ?). It has been argued that the stereotyped signals that the brain uses are symbols that do not resemble in any way the external world they represent ((Nicholls et al., 1992), chapter 1). Psycho-physical experiments (Cooper and Shepard, 1984), on the other hand, point to processing in the CNS that is governed by laws of physics (Deutsh and Deutsch, 1993; Wolpert et al., 1995). Singer (Singer, 2000) states that "neurons encode information solely by varying their discharge rate, or additional information is contained in the precise temporal relationship between the discharges in a population of neurons." Hebb (1949) states that, " information storage involves an increase in the area of contact between the preand post-synaptic neuron". Horn (1962) states that "information storage involves an increase in the number of receptors in the post-synaptic membrane, and an increase in the neurotransmitter release."

System theoretical concepts have been used to describe timed events in single neurons or designing delay compensating neural elements. Arbib (Arbib, 1995), page 53 discusses leaky integrators as more realistic physical models of neurons. In Arbib's view, a neuron circuit with a long time constant becomes an approximate integrator and, hence, a basic memory element.

Doeringer and Hogan (Doeringer and Hogan, 1998) have estimated the neural transmission delay in the spinal reflex loop of the upper extremity to be in the order of $30 \mathrm{~ms}$, with a transfer function that has a frequency range of zero to less than $2 \mathrm{~Hz}$. An inverse compensator is proposed for the delay - a form of predictor (Houk et al., 1981). Predictors are also discussed in ((Arbib et al., 98), page 62) and (Paulin, 1998). A single trajectory of force or motion (Kandel et al., 1991; Deutsh and Deutsch, 1993) can be stored in a tapped delay line (Kailath, 1984; Mayhan, 1984), compatible with the structure of short-term memory ((Deutsh and Deutsch, 1993; Eichenbaum, 2002), page 86 and chapter 9). The fading away of the information can be modeled as attenuation in a lossy delay line.

Still larger units of the CNS function can be envisioned that are involved in signal generation and processing. Time scaling and piecewise linear signals are examples. Time scaling involves producing a slower or faster movement. Kornhuber (Kornhuber, 1971; Brooks, 1986) states "There is an additional ability of hand movement as compared to eye movement. A ramp function smooths non-ballistic movements of the hand as well as arms and legs." Kornhuber attributes the source of ramp signals to the basal ganglia based on the following argument. The eyes take movement commands directly from the cerebellum. The appendages take commands from both the basal ganglia and the cerebellum via the motor cortex. The additional smooth movement capability must come from the basal ganglia.

\subsection{Pattern Generation}

Timed events are involved when the CNS has to relate internal decisions to external events. Timing (Gribova et al., 2002; Macar et al., 2004; Nobre and Reilly, 2004) relative to an external cue or alarm, could involve associative learning (Kandel et al., 1991, Chapter 65). Timing is also involved in mechanisms of attention (Evarts et al., 1984), or preparation for a movement or in a motor set (Kornhuber, 1971; Brooks, 1986). Foster and Wilson (Foster and Wilson, 2006) found that while rats rested after running a maze, their brains replayed the movements in reverse. The idea of replaying events in reverse could explain the synchronization of internal action with external events.

There are cases in CNS where different sensory neurons fire in synchrony (Goddard et al., 1992; Kandel et al., 1991), chapter 30. A simple example of this is the eye-blink experiment. In this experiment (Eichenbaum, 2002; Grethe and Thompson, 2003), blowing a puff of air in a rabbit's eye will cause the rabbit to blink (Grethe and Thompson, 2003). Grethe and Thompson (Grethe and Thompson, 2003) review and compare models of this synchronization. Tapped delay lines of different lengths (Miall, 1995; N. and Wang, 1995; Wolpert et al., 1995]) were proposed. Artificial neural networks (ANN) can mimic the coincidence model of Buhusi and Meck (2005), Granule cells begin to oscillate at the start of the conditioned stimulus, and then the outputs of these granule cells are recorded at the time of the stimulus. Nicholls et al. (p. 552 ) discuss, for medullary neurons that generate the respiratory rhythm in neonatal opossum, the location of synapses and the incremental time delays that are important in temporal processing and signal shaping.

Simultaneous patterns may have to be recalled from storage or from pattern generators ((Levitan and Kaczmarek, 1991), chapter 17). Two alternative schemes to generate patterns are by linear filters and neural oscillators (Bay and Hemami, 1987; Wang and Alkon, 1993, Kaczmarek and Levitan, 1987). The transient outward current in the bag cell neuron is shown to contain one exponential function of time with time constant of $76 \mathrm{~ms}$ and, under the influence of cyclic AMP, to contain a second exponential of a much faster decay and, hence, a rationale for a second-order system. Neuro-transmitters change the shape of an action potential ((Levitan and Kaczmarek, 1991), page 249), suggesting the possibility of filtering and processing mechanisms that are implicit in this case: the narrowing of the action potential pulse in the dorsal root ganglion of a chick.

Patterns are involved in voluntary movements and also in two elicited maneuvers when the base is disturbed (McCollum et al., 1984; Szturm and Fallang, 1998) or the subject slips or slides (Pai, 1999). Iqbal and Roy (Iqbal and Roy, 2009) present a neuro-musculoskeletal system that could serve as a test bed here. Simpler systems also have been utilized (Hemami et al., 2006). 
The exploration of centrally issued signals is a major study in biological systems, and here it implies a systems-oriented study of neural manipulations of external and internal events in bio-mechanical systems. These timed events take place in the motor system of the central nervous system (CNS) in living systems and in robotic and man-made systems. In the motor system, timed events may be constructed in real time or made, alternatively, available in short-term memory (STM) for further processing. They may be sequenced to construct longer output events. Coordination with external events, storage, recall, delaying or advancing in time, synchronizing, time scaling and inverting events in time are other examples of such CNS processing. The hope is to motivate future experimental work in identifying larger processing units of the CNS and their precise description in a system theoretical framework.

\subsection{Processing}

CNS computations involve procedural memory and stack processing. Models of procedural memory and stack processing are reviewed here.

\subsection{Procedural Memory}

Three implementations for procedural memory are described here.

1. Sequential triggering of signals and chaining is one way to generate commands for complex movements (Massey et al., 1986). We consider a very simple case here. The first movement starts as output of a block. When the first movement is complete, a trigger starts the second movement and so on. Each block of the sequence triggers the next until the sequence of movements is complete. Asynchronous "one-shot" circuits without a clock are utilized ((Berlin, 1976), Fig 5.11).

2. In delay line memory, capacity and information loss are two characteristic attributes, and the implementation is also feasible by neural networks (Wang and Alkon, 1993). Newer information will push out older information and short-term memory experiences loss of information over time. A short-term memory, with the above two attributes, can be implemented by a delay line structure or by an artificial neural network (ANN).

3. The third implementation is ramp-activated. By changing the slope of the ramp signal, the duration of the event is changed . A slope larger than unity produces a compressed signal and a slope less than unity stretches the signal linearly in time.

\subsection{LIFO Stacks}

The conditioned response seems to be coordinated in the lateral cerebellum (Grethe and Thompson, 2003). The method here maps time uniformly to locations and intervals of time to distances in a stack. For simplicity, we do not consider the number of trials needed to learn and optimize the conditioned response. We only present the final processing that has been perfected and executed successfully. The process is implemented here by three last-in first-out(LIFO) stacks. The information is pushed down the stack upon command. The information here is a short auxiliary pulse (as a function of time). The three stacks are called, respectively, the stimulus, the processor and the action stack. The stimulus stack keeps track of and measures the time transpired between the warning signal and the external action, such as blowing a puff of air. This means the detection of the external action stops the pushing operation in the stack. The processor stack is capable of the same as the stimulus stack, but can be stopped at any time to hold the propagating short pulse at a particular location (time) in the stack. All the storage locations of these two stacks are also accessible in parallel so that the content of the corresponding pairs of memory locations can be compared at any time. The third LIFO stack keeps track of the total needed time for closing the eyes from the moment of the intention to the time the eyes are fully closed.

The abstracted version of what the rabbit in the aforementioned experiment may have learned is executed in three steps:

1. A warning signal triggers the pushing operation in both the stimulus and the processor stacks. This means two pulses propagate down these stacks. An action, such as the blowing of a puff of air, stops the pushing action of both stacks. Let this measured time interval be $\mathrm{t} 1$.

2. The intention to close the eyes triggers the pushing action in the action stack. The closing of the eyes stops pushing in the second stack. Let this total measured time be $\mathrm{t} 2$.

3. All three stacks start to pop simultaneously, until the action stack is empty. At this time, the processor stack is frozen, that is, it is retaining one pulse at location $\mathrm{t} 1-\mathrm{t} 2$ from the top of the stack. The stimulus stack is cleared.

There are three steps for timely closing of the eyes: 
1. The stimulus stack starts pushing after a warning signal is issued.

2. At location (time) $\mathrm{t} 1-\mathrm{t} 2$, both stacks have the resident short pulse and this coincidence triggers the intention to close the eyes.

3. The eyes close at the right time.

Similar stacks can be used to create a piecewise triangular function of time from the first half, i.e., the ramp upward part of the signal. The first half is pushed into a LIFO stack. The ramp downward (i.e., the second half of the pulse) is extracted from the stack by popping during the second half time interval.

A number of important items are presented here that may be relevant to human eye movement. However, physical or anatomical evidence is not available for what is stated here. Two reasons for the discussions here are motivation for future research and relevance to the construction of artificial eyes in the future.

In this section, two movements are considered in some detail: smooth pursuit and ballistic fast movements (Hannaford and Lehman, 1986; Hemami and Barin, 2017; Kandel et al., 1991). The eye saccades (Hannaford and Stark, 1985) are interpreted as fast movements of the eyes, similar to fast movement of the head (Hannaford and Stark, 1984). At the same time, eye movements are different than the head (or body) movements in several ways:

- no external and or variable loading,

- small inertias, and friction, and

- small amplitudes and small excursions.

As a consequence, and to a first order of approximation, one can assume that the eye transients are fast and negligible and can be ignored. This means the system follows central inputs accurately. Therefore, a major component of central inputs is the design of kinematic signals that are responsible. A point of hope here is the extensive availability of experimental, medical and diseased (Bahill and Stark, ) data available from internal and external injuries, handicaps and natural failures.

Before presenting the above issues, it is important to establish that the dynamics of the eye, in terms of stability and control exerted by the muscular system, are equivalent to the dynamics of the muscular system of the head. This subject merits a comprehensive analysis and is postponed for a separate effort.

A second issue, in this regard, is the physical geometry and attachment of the eyeball muscular insertions and origins and their relevance to specific motions and eye trajectories. Higher-level neural circuits are always involved. Another important issue from system theoretical and neural points of view is the issue of constraints. Due to the attachment constraints, the motion of the eye in the RCS or LCS is coupled. For specific motions along the different axes there is need for decoupling or, alternatively, projecting the desired motion as combination of other physically available motions.

\section{Discussion and Conclusions}

This paper has presented a very simple model of the ocular system involved primarily in the motion of the eyeballs and not in the sensory capacity of the eyes in the vision system. The system appears to function primarily as a feed-forward system with minimal feedback. The model is not adequate for extensive and precise processing afforded by the CNS. The involvement of the vestibular system in collaborative and competitive head and eyeball motion points to some other remarkable abilities of the CNS. With two sequential integrations by long term potentiation LTP's the velocities and position angles of the head also are available as inputs to the ocular system. Also, stored saccade trajectories of the head can be used as central models for the reference inputs for eye saccades.

At the same time, movement is one of the simplest abilities (common to other species) of the human brain. It is a challenge to develop comprehensive mathematical tools that function exactly as the natural components of the CNS. These tools would collaborate on the physical, mechanical, chemical, neural and physiological subsystems of the human body by common tools of mathematics and simulation apparatus.

\section{Acknowledgment}

The authors are indebted to Professors Joel Johnson and H. El Gamal, two Chairs of the Department of Electrical and Computer Engineering at The Ohio State University for encouragement, and support. The authors are grateful to all the staff members of The Old Worthington Library, specially Christin Demay and Andrew Cline for all the help. 


\section{Appendix A}

The approximate coordinates of nine important points in the HCS of an average size human are listed here. All are given in meters. The center of the coordinate system is assumed to be at the connection point of the head to the neck. A visual image of the ocular muscles is available in Grant's Atlas of Anatomy, Figures 522 and 523.

- The center of gravity of the head:

$$
\text { COGH }=[0.0,0.0,0.04]^{T}
$$

- The center of the right eyeball:

$$
C C R E=[0.08,-0.0325,0.08]^{T}
$$

- Center of the left eyeball:

$$
C C L E=[0.08,0.0325,0.08]^{T}
$$

- Common origins of the right eye recti muscles:

$$
\text { ORRM }=[0.054,-0.0325,0.08]^{T}
$$

- Common origins of the left eye recti muscles:

$$
\text { CRIM }=[0.054,0.0325,0.08]^{T}
$$

- Trochlear of the right eyeball:

$$
C T R E=[0.09,-0.0165,0.096]^{T}
$$

- Trochlear of the left eyeball:

$$
C T I E=[0.09,0.0165,0.096]^{T}
$$

- Origin of the inferior oblique of the right eye:

$$
\text { COIOR }=[0.076,-0.03,0.068]^{T}
$$

- Origin of the inferior oblique of the left eye:

$$
\text { COIOL }=\left[0.076,0.03,0.068 l^{T}\right.
$$




\section{References}

Angevine Jr, J. B. and Cotman, C. W. (1981). Principles of Neuroanatomy. Oxford University Press, Oxford, second edition.

Arbib, M., Erdi, P., and Szentagothai, J. (98). Neural Organization, Structure, Function, and Dynamics. A Bradford Book, The MIT Press, Cambridge, Mass.

Arbib, M. A., editor (1995). The Handbook of Brain Theory and Neural Networks. The M.I.T. Press, Cambridge, Mass.

Asanuma, H. and Rosen, I. (1972). Topographical organization of cortical efferent zones projecting to distal forelimb muscles in the monkey. Experimental Brain Res., pages 243-256.

Bahill, A. T. and Stark, L. Overlapping saccades and glissades are produced by fatigue in the saccadic eye movement system. Experimental Neurology, (48):95-105.

Basmajian, J. (1978). Muscles Alive. The Williams and Wilkins Company.

Bay, J. S. and Hemami, H. (1987). Modeling of a neural pattern generator with coupled nonlinear oscillators. IEEE Trans. Biomedical Engineeing, 34:297- 306.

Becker, W. and Juergens, R. (1975). Saccade relations to double-step stimuli: Evidence for model feedback and continuous information uptake.

Berlin, H. M. (1976). The 555 Timer Applications Sourcebook, with Experiments. Howard W Sams and Co, Inc., Indianapolis.

Brooks, V. (1986). The neural basis of motor control. Oxford University Press.

Buhusi, C. V. and Meck, W. H. (2005). What makes us tick? functional and neural mechanisms of interval timing. Nature Reviews Neuroscience, 6:755-765.

Collins, C. C. (1975). The human oculomotor control system. In Basic Mechanisms of Ocular Motility and Their Clinical Implications, pages 145 - 180. Pergamon Press, Oxford and New York.

Cooper, L. A. and Shepard, R. N. (1984). Turning something over in the mind. Scientific American, 251(6):106 - 114.

Deutsh, S. and Deutsch, A. (1993). Understanding the Nervous System. IEEE Press., first edition.

Doeringer, J. A. and Hogan, N. (1998). Serial processing in human movement production. In Neural Networks, pages 1345-1356. Pergammon.

Eichenbaum, H. (2002). The Cognitive Neuroscience of Memory. Oxford University Press, Inc., first edition.

Evarts, E., Bizzi, E., Burke, R., DeLong, M., and Thach, Jr., W. (1971). Central control of movement. Neuroscience Res. Prog. Bulletin, pages $1-170$.

Evarts, E., Shinoda, Y., and Wise, S. (1984). Neurophysiological Approaches to Higher Brain Functions. John Wiley and Sons.

Foster, D. J. and Wilson, M. A. (2006). Reverse replay of behavioral sequences in hippocampal place cells during the awake state. Nature, 444:680 - 683 .

Goddard, R., Boyer, K., and Hemami, H. (1992). Collision strategies for robotic retreat and resistance. In Tzafestas, S., editor, Microprocessors in Robotic and Manufacturing Systems, chapter 8, pages 177-215. Kluwer Academic Publishers.

Goldberg, M., Eggers, H. M., and Gouras, P. The ocular motor system.

Gray, H., editor (1977). Gray's Anatomy. Bounty, first edition.

Grethe, J. S. and Thompson, R. F. (2003). Cerebellum and conditioning. In Arbib, M., editor, The Handbook of Brain Theory and Neural Networks. The M.I.T. Press, Cambridge, Mass., second edition. 
Gribova, A., Donchin, O., Bergman, H., E., V., and de Oliveira, S. C. (2002). Timing of bimanual movement in human and nonhuman primates in relation to neuronal activity in primary motor cortex and supplementary motor area. Experimental Brain Research, 146.

Hannaford, B. and Lehman, S. (1986). Short time fourier analysis of the electromyogram: fast movements and constant contraction. IEEE Transactions on Biomedical Engineering., (12):1173-1181.

Hannaford, B. and Stark, L. (1984). Control strategies for neurologically ballistic movements. In Proceeding, Annual International Conference of the IEEE Engineering in Medicine and Biology, pages 640 - 655. IEEE, New York.

Hannaford, B. and Stark, L. (1985). Roles of the elements of the tri-phasic control signal. Experimental Neurology, (3):619-634.

Hebb, D. (1949). The Organization of Behavior. John Wiley and Sons, first edition.

Hemami, H. (1982). A state space model for interconnected rigid bodies. IEEE Trans. on Automatic Control, (2):376-382.

Hemami, H. (2002a). A general framework for rigid body dynamics, stability and control. Journal of Dynamic Systems, Measurement and Control, pages 241-251.

Hemami, H. (2002b). A general framework for rigid body dynamics, stability and control. J. Dynamic Systems, Measurement and Control, 124:241-251.

Hemami, H. (2003). Evolutionary trends in rigid body dynamics. Computer Methods in Applied Mechanical Engineering, pages $635-654$.

Hemami, H. (2017). A model of early motivational states and their uses. Mech. Engineering Research, (1):31-47. Doi: 105539/mer.v7n1p31.

Hemami, H. and Barin, K. (2017). Preliminary design of a generalized vestibular sensory system. International J. of Astronautics and Aeronautical Engineering, 2:003:1 -11.

Hemami, H., Barin, K., and Pai, Y.-C. (2006). Quantitative analysis of a sagittal biped under platform disturbance. IEEE Trans. Neural Systems and rehabilitation Engineering, (4):470-480.

Hemami, H. and Dariush, B. (2016). Neural and spinal modules in implementation of a simple ballistic movement. Software Engineering and Applications, Special issue: Robotics and Automation, 9(7):326-345. paper number: 9302206, [336].

Hemami, H. and Dinneen, J. (1995). Simple direction-dependent rhythmic movement and partial somesthesis of a marionette. IEEE Transactions on Systems, Man and Cybernetics, part B, (11):1491-1501.

Hemami, H. and Hemami, M. (2016). State space models and examples for computational models of human movement. Mechanical Engineering Research, 6(1):46 -65.

Hemami, H. and Katbab, A. (1982). Constrained inverted pendulum for evaluating upright stability. Journal of Dynamic Systems, Measurement and Control, pages 343-349.

Hemami, H., Khosravi-Sichani, B., and Barin, K. (2016a). Airborne and landing phases of a simplified back somersault movement. Computer and Electrical Engineering, 53:1-12.

Hemami, H. and Moussavi, Z. M. (2014). A model of the basal ganglia in voluntary movement and postural reactions. Computer Methods in Biomechanics and Biomedical Engineering, 20(1):1432-1446. DOI: 10:1080/1055842.2012.751983.

Hemami, H., Tarr, E., Li, B., Krishnamurthy, A., Clymer, B., and Darius, B. (2016b). Towards a cybernetic models of human movement. Mechanical Engineering Research, 6(1):1-18.

Hemami, H. and Utkin, V. (2002). On the dynamics and lyapunov stability of constrained and imbedded rigid bodies. International Journal of Control, 75(6):408 - 420.

Hemami, H. and Wyman, B. F. (2012). A simple strategy for jumping straight up. Math. Biosciences, pages $28-37$.

Horn, G. (1962). Some neural correlates of perception. In Carthy, J. and Duddington, C. L., editors, Viewpoints in Biology, pages 242-285. Butterworth. 
Houk, J., Crago, P., and Rymer, W. (1981). Function of the spindle dynamics response in stiffness regulation - a predictive mechanism provided by nonlinear feedback. In Taylor, H. and Prochazka, A., editors, Muscle receptors and Movement, pages 299 - 309. MacMillan.

Howard, I. P. (1982). Human Visual Orientation. John Wiley and Sons, New York.

Humphrey, L. R. (2009). Modeling of the Foot, Toes and Vestibular System in Human Balance. PhD thesis, The Ohio State University, Columbus, Ohio.

Iqbal, K. and Roy, A. (2009). A novel theoretical framework for the dynamic stability analysis, movement control, and trajectory generation in a multisegment biomechanical model. J. of Biomechnical Eng, Trans. ASME, 131(1):002013.

Jalics, L., Hemami, H., Clymer, B., and Groff, A. (1997). Rocking, tapping, and stepping, a prelude to dance. Autonomous Robots, 4:227-242.

Kailath, T. (1984). Linear Systems. Prentice Hall Inc., Englewood Cliffs, New Jersey.

Kandel, E., Schwarrtz, J., and Jessell, T. (1991). Principles of Neural Science. Elsevir, third edition.

Khalil, H. (1996). Nonlinear Systems. Prentice Hall.

Kokotovic, P. and Arcak, M. (2000). Activation of nonlinear feedback concepts. In System Theory, Modeling, analysis and Control, pages 379-389. Kluver Academic Publishers.

Kokotovic, P. V., Khalil, H. K., and O’Reilly, J. (1986). Singular Perturbation Methods in Control. Academic Press.

Kornhuber, H. H. (1971). Motor functions of cerebellum and basal ganglia: The cerebellocortical saccadic (ballistic) clock, the cerebellonuclear hold regulator, and the basal ganglia ramp (voluntary speed smooth movement) generator. Kybernetik, 8 Bd., Heft 4, pages 157-162.

Levitan, I. B. and Kaczmarek, L. (1991). The Neuron, Cell and Molecular Biology. Oxford Univ. Press.

Macar, F., Anton, J., Bonnet, M., and Vidal, F. (2004). Timing functions of the supplementary motor area: an event-related fmri study. Cognitive Brain Research, 21.

Massey, J., Schwartz, A., and Georgopoulos, A. (1986). On information processing and performing a movement sequence. Experimental Brain Research, series 15.

Mayhan, R. (1984). Discrete-Time and Continuous-Time Linear Systems. Addison Wesley, Reading, Mass.

McCollum, G., Horak, F., and Nashner, L. (1984). Parsimony in neural calculations for postural movements. In et al., B., editor, Cerebellar Functions, pages 52 - 66. Springer-Verlag, Berlin.

Miall, R. (1995). Motor control, biological and theoretical. In Arbib, M., editor, The Handbook of Brain Theory and Neural Networks, pages 597 -600. The M.I.T. Press.

Nicholls, J., Martin, A., and Wallace, B. (1992). From Neuron to Brain. SinauerAssociates, Inc., third edition.

Nobre, A. and Reilly, J. (2004). Time is of the essence. Trends in Cognitive Sciences, 8.

Pai, Y.-C. (1999). Induced limb collapse in a sudden slip during termination of sit to stand. J. Biomechanics, pages 1377-1382.

Paulin, M. (1998). A method for analyzing neural computation using receptive fields in state space. Neural Networks, pages 1219,1228 .

Rosen, I. and Asanuma, H. (1972). Peripheral afferent inputs to the forelimb area of the monkey motor cortex. Experimental Brain Res., (4):150-157.

Singer, W. (2000). Response synchronization, a neural code for relatedness. In J., B. J., editor, Brain, Perception, Memory, pages 35-48. Oxford University Press.

Szentagothai, J. and Arbib, M. (1974). Conceptual Models of Neural Organization, NPR Bulletin, volume 12 , No 3. Neurosciences Research Program. 
Szturm, T. and Fallang, B. (1998). Effects of varying acceleration of platform translation and toes-up rotations on the pattern and magnitude of balance reactions in humans. Journal of Vestibular Research, 8(5):381-397.

Utkin, V. (2009). Linear System Theory. McGraw Hill Book Co, Inc.

Wang, L. and Alkon, D. L. (1993). Artificial Neural Networks. IEEE Computer Society.

Winters, J. (1990). Hill-based muscle models: A system engineering perspective. In Multiple Muscle Systems: Biomechanics and Movement Organization, pages 69 - 93. Springer Verlag, New York.

Wolpert, D., Ghahramani, Z., and Jordan, M. (1995). An internal model for sensorimotor integration. Science, New Series, (5232):1880-1882.

Wongchaisuwat, C., Hemami, H., and Buchner, H. (84). Sliding and rolling at natural joints. ASME Trans.,Journal ofBiomechanical Engineering, 106:368 -375.

\section{Copyrights}

Copyright for this article is retained by the author(s), with first publication rights granted to the journal.

This is an open-access article distributed under the terms and conditions of the Creative Commons Attribution license (http://creativecommons.org/licenses/by/4.0/). 